\title{
A space-charge-neutralizing plasma for beam drift compression
}

\author{
P. K. Roy ${ }^{1}$, P. A. Seidl ${ }^{1}$, A. Anders ${ }^{1}$, F. M. Bieniosek ${ }^{1}$, J. E. Coleman ${ }^{1,2}$, E. P. Gilson ${ }^{3}$, W. Greenway ${ }^{1}$, D. P. \\ Grote $^{4}$, J. Y. Jung ${ }^{1}$, M. Leitner ${ }^{1}$, S. M. Lidia ${ }^{1}$, B. G. Logan ${ }^{1}$, A. B. Sefkow ${ }^{3,5}$, W. L. Waldron ${ }^{1}$, and D. R. \\ Welch $^{6}$ \\ ${ }^{1}$ Lawrence Berkeley National Laboratory, Berekeley, CA 94720, USA \\ ${ }^{2}$ Department of Nuclear Engineering, University of California, Berkeley, CA 94720, USA \\ ${ }^{3}$ Princeton Plasma Physics Laboratory, Princeton, NJ 08543, USA \\ ${ }^{4}$ Lawrence Livermore National Laboratory, Livermore, CA 94550, USA \\ ${ }^{5}$ Sandia National Laboratories, Albuquerque, NM 87185 \\ ${ }^{6}$ Voss Scientific, Albuquerque, NM 87108, USA
}

\begin{abstract}
Simultaneous radial focusing and longitudinal compression of intense ion beams are being studied to heat matter to the warm dense matter, or strongly coupled plasma regime. Higher compression ratios can be achieved if the beam compression takes place in a plasma- filled drift region in which the space-charge forces of the ion beam are neutralized. Recently, a system of four cathodic arc plasma sources has been fabricated and the axial plasma density has been measured. A movable plasma probe array has been developed to measure the radial and axial plasma distribution inside and outside of a $\sim 10 \mathrm{~cm}$ long final focus solenoid (FFS). Measured data show that the plasma forms a thin column of diameter $\sim 5 \mathrm{~mm}$ along the solenoid axis when the FFS is powered with an $8 \mathrm{~T}$ field. Measured plasma density of $\geq 1 \times 10^{13} \mathrm{~cm}^{-3}$ meets the challenge of $n_{p} / Z n_{b}>1$, where $n_{p}$ and $n_{b}$ are the plasma and ion beam density, respectively, and $Z$ is the mean ion charge state of the plasma ions.

Keywords: Beam; ion; neutralization; compression; plasma;plasma probe; plasma density;
\end{abstract}




\section{Introduction}

Intense ion beams of low kinetic energy offer an attractive approach to heating dense matter uniformly to extreme conditions, because their energy deposition is nearly classical and shock-free. Ion beams have the ability to heat target materials (conductors, insulators, foams, and powders) with high repetition rates. High energy density physics and heavy-ion-driven inertial fusion require the simultaneous transverse and longitudinal compression of an ion beam to achieve high intensities. For example, a beam of $\sim 200 \mathrm{~A} \mathrm{(23} \mathrm{MeV}$ $\mathrm{Na}^{+}$) with a 1-mm focal spot radius and pulse length of $\sim 1$ ns would be suitable as a driver for Warm Dense Matter experiments. These beam spot sizes and pulse lengths are achievable with beam neutralization [1-4] and longitudinal compression [5-14] in background plasma.

In beam neutralization, electrons from a plasma or external source are entrained by the beam and neutralize the space charge sufficiently such that the pulse focuses on the target in a nearly ballistic manner to a small spot, limited only by longitudinal and transverse emittance. Typically, $\mathrm{n}_{\mathrm{p}} / \mathrm{Zn}_{\mathrm{b}}>1$, where $\mathrm{n}_{\mathrm{p}}$ is the plasma density, and $n_{b}$ and $Z$ are the ion beam density and mean charge state, respectively. Figure 1 shows an experimental example of beam neutralization [3]. It has been demonstrated that when a $300 \mathrm{keV}, 25 \mathrm{~mA} \mathrm{~K}{ }^{+}$beam passed through vacuum, the beam radius at the nominal $1 \mathrm{~m}$ focal distance was $14.7 \mathrm{~mm}$ at $1 \sigma$ radius. The same beam, when passed through a plasma plug, a conjunction of two plasma jets, using two filtered cathodic arc sources (FCAPS) [15-16], exhibited a beam $1 \sigma$ radius that decreased to $\sim 1.3 \mathrm{~mm}$.

For longitudinal compression, an induction bunching module (IBM) produces a head-to-tail velocity ramp that longitudinally compresses the beam for a short pulse length. Figure 2 shows such an example [10], where a $300 \mathrm{keV}, 25 \mathrm{~mA} \mathrm{~K}{ }^{+}$beam was longitudinally compressed by the IBM, creating an enhanced beam peak current density of $>50$ with pulse a duration of about $2.5 \mathrm{~ns}$. In this example, the beam spot size at the focal plane was larger than the neutralized beam spot, as mentioned in the first example. This was due to a lack of sufficient plasma density that was needed to neutralize space charge of the highly compressed ( $>50$ fold) beam. In this example, plasma, from the two FCAPS, was transported upstream to the IBM using a solenoid coil. The initial plan was to use a plasma column, known as a Ferroelectric Plasma Source (FEPS) [17] that ejects plasma in the 
radial direction, and use of the two FCAPS at the downstream end. However, the FEPS was under characterization in a separate bench test, and therefore the two FCAPS system was used, including a plasma channel made of a long solenoid coil.

The above two experimental examples demonstrate that sufficient plasma is essential for radial and longitudinal compression. The beam intensity, immediately after the longitudinal compression, is significantly higher than the uncompressed beam, illustrating that a higher plasma density is required to maintain a small beam spot size.

In recent years, much attention has been given to the simultaneous radial and longitudinal compression experiments [11-14], in addition to suggestions that a higher plasma density channel is crucial to use an ion beam as a driver to study target properties in a campaign on warm dense matter (WDM) studies [18]. Initially the target would be a thin self-supporting gold or aluminium metallic foil, mounted on a glass or sapphire substrate. A simulation [13] suggested that the compressed beam pulse reaches $10^{12} \mathrm{~cm}^{-3}$ peak density vs. a beam density of $\sim 2 \times 10^{8} \mathrm{~cm}^{-3}$ entering the IBM gap. Also, the simulation predicted the longitudinal compression ratio should peak at 120 with a transverse spot size $a_{s}=1.3 \mathrm{~mm}$ (edge radius). Thus, a target placed at the focus would receive peak deposition energy of $\geq 0.035 \mathrm{~J} / \mathrm{cm}^{2}$ for a $300 \mathrm{keV} 26 \mathrm{~mA}$ beam. Practical limitations of the beam dynamics include emittance, chromatic aberration, and achieving high plasma electron density near the focal plane. In parallel, use of a strong beam focusing field had also been suggested [11] to focus the intense beam to the target. A new final focus solenoid (FFS) $10 \mathrm{~cm}$ long with an $8 \mathrm{~T}$ field is considered in such a way that the FFS fringe field is to be used to carry plasma from the downstream FCAPS systems, in parallel of intense beam focusing to the target plane. In order to increase the plasma density, the FCAPS system has been replaced by fabricating a system of four cathodic arc plasma source (CAPS) system. A radial plasma probe has been developed to measure the radial plasma distribution.

In this article, Sec. II describes the final focus plasma channel. Sec. III describes the fabrication of a fourCAP system for a higher plasma density. Section IV represents development of a radial plasma probe, and measurements of radial and axial plasma density. Finally, a summary of key results is presented in Sec. V. 
HIF08,PKROY

\section{A final focus plasma channel}

The final focus plasma channel consists of either a two-FCAPS or a four-CAP system located in the target chamber; the $10 \mathrm{~cm}$ long final focus solenoid (FFS); and a long ferroelectric plasma source (FEPS). The physics of the FCAPS and FEPS are described elsewhere [15-17]. Here we describe the FFS and the axial plasma density distribution when the FES is operated. The effectiveness of the final focus plasma channel was tested in a beam compression experiment.

\subsection{Final focus Solenoid (FFS)}

Figure 3(a) shows a sketch of the pulsed FFS. The solenoid coil ([Fig. 3(b)] has 32 turns (8 turns/layer) with the inner and outer diameters of the winding pack at $5.1 \mathrm{~cm}$ and $6.7 \mathrm{~cm}$, respectively. Table 1 summarizes the main magnet parameters. In order to limit eddy currents, the solenoid winding consists of Litz wire wound on a non-conductive G-10 tube. The winding pack was inserted into a stainless steel housing and it was potted with an electrically insulating, thermally conducting highly viscous epoxy (Cool-Poly ${ }^{\circ}$ D1202) (The single strand of the Litz wire can be potted.). The eight-Tesla final-focus solenoid magnet with $837-\mu$ s pulses and a peak current of $21.7 \mathrm{kA}$, requires more than $1.5 \mathrm{~kJ}$ per pulse, which necessitates active cooling. The magnet is cooled by compressed air and water flowing through cooling channels [Fig. 3(c)]. The magnetic stored energy is $4.5 \mathrm{~kJ}$ for the physical length $(9 \mathrm{~cm})$ of the solenoid. The cooling channel housing is also used for the protection from the mechanical stress generated by the magnetic stored energy. Figure 3(d) shows an example of the field profile measured during initial tests using $6.5 \mathrm{kA}$. The solenoid has an operating repetition rate of $\approx 0.05 \mathrm{~Hz}$. The magnet cooling system keeps the solenoid coil at room temperature $\left(T \leq 25^{\circ} \mathrm{C}\right)$ for 30 minutes of operation with a 0.040 $\mathrm{Hz}$ pulse rate, when operated with an eight-Tesla field.

If the Litz wire (Fig. 3b) was not chosen to reduce the eddy current in the conductors, the deformation of the magnetic field due to eddy currents might be a serious issue.

\subsection{Axial plasma density}

Figure 4(a) shows a sketch of the FFS assembly with the two FCAPS systems, which are also used for NTX [3] and NDCX-1[10] experiments. The FCAPS system was formed by two pulsed aluminium cathodic arc 
sources. Each source was equipped with a $30^{\circ}$ macroparticle filter oriented towards the FFS providing a flow of fully ionized aluminium plasma. A single collector Langmuir probe was driven through the beam axis from the upstream end of the FFS to the target plane. The distances between the target plane (here, the FCAP source filter apertures), and the downstream-end and the center of the FFS were $11.7 \mathrm{~cm}$ and $18.27 \mathrm{~cm}$, respectively. The original long, thin Langmuir probe (with $0.19 \mathrm{~mm}$ radius, and $11 \mathrm{~mm}$ long) was modified by a thumbtack-shaped -flat probe (with a $3.2 \mathrm{~mm}$ radius) to represent an ion collection surface normal to the magnetic field. The pin probe $\left(11.94 \mathrm{~mm}^{2}\right)$ and the backside of the Langmuir probe tip were covered using insulating kapton tape. The probe was operated with an applied voltage of $-90 \mathrm{~V}$ to $-95 \mathrm{~V}$ to collect a signal in the ion saturation mode. The plasma ion current was measured between the solenoid and the target plane, where the beam density would be the highest. The maximum peak plasma ion current of $9.9 \mathrm{~mA}$ was measured between the gap of the target plane and the FFS, when the FFS was not powered. The measured plasma ion current was as high as $45 \mathrm{~mA}$ when the FFS was powered with a $4 \mathrm{~T}$ field. Measured data was plotted as an axial plasma density distribution, which depends on the mean plasma ion velocity distribution. In principle, the plasma density is expressed as, $n_{p}=I_{p} /\left(A_{c} v_{p} q Z\right)$, where $I_{p}$ is the plasma current, $A_{c}$ is the area of a collector, $v_{p}$ is the plasma ion velocity, and $q$ is the charge of a single particle with the number of its charge state, $Z$. The average velocity of injected plasma was varied roughly between 1.4 to $6 \mathrm{~cm} / \mu \mathrm{s}$ as measured using the time of flight (TOF) method (velocity=distance/time). The jitter was on the order of $5 \mu \mathrm{s}$, and over a $10 \mathrm{~cm}$ time-of-flight span this corresponds to a $2 \mathrm{~cm} / \mu \mathrm{s}$ uncertainty. A plasma velocity in the presence of the solenoid was obtained using LSP [19] simulations with initial velocities of 1.5 $\mathrm{cm} / \mu \mathrm{s}$ and $6 \mathrm{~cm} / \mu \mathrm{s}$. A maximum plasma density of $2.8 \times 10^{12} \mathrm{~cm}^{-3}$ was achieved between the gap of the FFS and the target plan, where the plasma ion velocity was lower due to the FFS field. This density was reduced to $1.3 \times 10^{11} \mathrm{~cm}^{-3}$ at the same plane when the FFS was not powered. The data also shows that density was decreased as velocity increased at the upstream end of the FFS, but the density was higher $\left(\sim 2.5 \times 10^{11} \mathrm{~cm}^{-3}\right)$ than the density without the FFS field $\left(\sim 10^{10} \mathrm{~cm}^{-3}\right)$.

Some plasma particles were controlled by the FFS field, and enhanced the plasma density near the target area. Data shows that plasma density is not sufficiently high inside the solenoid even with the FFS powered. This may be due to the orientation of the plasma sources and their filters. Also, plasma was focused to the beam axis with $30^{\circ}$ bending of the filter. Much plasma $(>70 \%)$ is lost in the filter. Therefore, it was planned to further 
reduce the angle of the source relative to the beam axis, and to straighten the filter in order to reduce the plasma losses inside the filter and enhance the plasma density inside the FFS.

\subsection{Integration of the FFS and the two-FCAPS for simultaneous transverse and longitudinal compression}

Figure 5(a) shows integration of the final focus solenoid assembly with the NDCX beam line to measure simultaneous transverse and longitudinal beam focusing at the target plane. A $\mathrm{K}^{+}$beam of $315 \mathrm{keV}$ and $28-\mathrm{mA}$ was focused by four new pulsed solenoid magnets [14] to control the beam envelope (beam radius and convergence angle). The solenoid replaced the four quadrupole magnets-previously used for the neutralized transport experiment (NTX) [3] and NDCX [10]. These were followed by an IBM for beam bunching, and an 85 $\mathrm{cm}$ long FEPS ( $9 \mathrm{kV}$ operating voltage) for primary beam neutralization, replacing the long solenoid plasma column used for the NDCX initial run [10]. These are integrated with the FFS system (5T or off) including the two FCAPS. The beam diagnostics were a multiple-pinhole Faraday cup, and a scintillator, the signal of which was detected using a gated camera through a quartz glass window ( $>90 \%$ transmission wavelength between 300 to $1000 \mathrm{~nm}$ ). A time-gated camera was also used to measure the beam optical profile and intensity.

Results of simultaneous transverse and longitudinal compressions using the FFS, the two-FCAPS system and the FEPS are shown in Fig. 5. FWHMs of $2.2 \mathrm{~mm}$ and $3.0 \mathrm{~mm}$ were measured when the FFS was powered with a $5 \mathrm{~T}$ field and not powered, respectively, as shown in Fig. 5(c). This result shows that the compressed beam spot is significantly decreased, and a higher plasma density may further decrease the focal beam spot size.

\section{The system of four cathodic arc plasma sources}

Two filtered cathodic arc plasma sources (FCAPS) were used to neutralize the beam space charge, providing a total plasma density of $\sim 10^{12} / \mathrm{cm}^{3}$ [Fig. 4(a)] near the target plane when the FFS was powered. The density was $<\sim 10^{11} / \mathrm{cm}^{3}$ when the FFS was not powered. To increase the plasma density, four new plasma sources were recently fabricated. Figures 6(a) and 6(b) show the new sources. The diameter of each of the aluminum cathodes is $0.64 \mathrm{~cm}$ [Fig. 6(c)]. Each of the four CAPS makes an angle of 50 degrees with the beam axis. The filters, which also serve as the arc discharge anode, are shorter and much less curved compared to the 
previous two FCAPS. A plasma filter for each of the cathodes is centered at a distance of $1.52 \mathrm{~cm}$ from the cathode center. The distance between a cathode surface to the first and last turns of the filter are 0.64 and $3.57 \mathrm{~cm}$, respectively. The distance between the center of a cathode and the center of a filter nozzle to the ion beam axis are $9.3 \mathrm{~cm}$, and $7.2 \mathrm{~cm}$, respectively. The new CAPS system was installed in the target chamber [Fig. 6(d)] along with the Langmuir probe and the FFS to measure the plasma density as a function of location. In this new configuration, the distance between the mid-plane of the FFS and the target plane was $19.3 \mathrm{~cm}$. All four CAPS were connected to a pulse forming network (PFN) capable of providing $\sim 800 \mathrm{~A}$ per plasma source.

As expected, the plasma ion current from the four CAPS system was higher than that from the twoFCAPS system. The cross sectional area of the Langmuir probe tip was reduced to $1.46 \mathrm{~mm}^{2}$ to avoid signal saturation. The axial plasma density distribution in the solenoid and between the solenoid and the NDCX focal plane (target plane) was measured, for magnetic field $\mathrm{B}=8 \mathrm{~T}(21 \mathrm{kA}$ peak current) and $\mathrm{B}=0$ in the final focus solenoid. Each of the four CAPS was operated with a discharge current of 770 Amperes.

Results show that the new four-CAPS system with the short straight filters provided $\sim 30$ times greater plasma density than the two-FCAPS system with the bent filters. Assuming a plasma velocity of $2 \times 10^{4} \mathrm{~m} / \mathrm{sec}$, the four-FCAPS system provided a plasma density $>10^{13} / \mathrm{cm}^{3}$.

\section{Measurement of the radial plasma profile}

The Langmuir probe has several drawbacks. It has a one to two $\mathrm{mm}$ driving dispersion within the $30 \mathrm{~cm}$ axial travelling distance, and the drive is along one axis only. In addition, the probe only covers an area of 1.46 $\mathrm{mm}^{2}$. Also, the cross sectional area might provide error when the covering kapton insulation (on the length of 11 $\mathrm{mm}$ tip mounting base and the back surface of the probe tip) burnt out with higher plasma discharges. Therefore, a new radial plasma probe was developed and axial and radial plasma density was measured.

\subsection{Design and development of a radial plasma probe array}

Figure 8(a) shows a photograph of the new plasma probe array for radial plasma density measurements. It is an assembly of 37 probes, made of copper wire (24 gauge). The diameter of each collector was $1.60 \mathrm{~mm}$, and the center-to-center distance between individual probes was $2.46 \mathrm{~mm}$. Each probe wire was housed inside an 
alumina tube. The outer and inner diameters of a tube were $2.46 \mathrm{~mm}$ and $1.64 \mathrm{~mm}$, respectively, and the length of a tube was $38.1 \mathrm{~mm}$. The seven collectors at the center line were covering a range of $1.7 \mathrm{~cm}$; this was the major diameter of the probe array. All of the collectors were formed into a bundle, and the bundle was housed into a Delrin tube. The front face of the complete array was diamond-polished in order to make each probe as similar as the next.

Each probe wire was terminated with a push-on connector for a wire leading to multi-pin feedthroughs. Because of the number of collectors, two multi-pin feedthroughs were used as shown in Fig. 8(b). An electric circuit was developed in such way that it was possible to bias and read out 10 collectors at a time. Figure 8(c) shows the circuit diagram for one collector. The drive unit of the probe was a $23.5 \mathrm{~cm}$ travel ball screw assembly. The precise $z$-motion position was established using a fine position monitor.

\subsection{Radial and axial plasma density}

We measured the plasma density distribution with the 37-collector probe array. Axial and radial plasma distribution measurements within the solenoid, and between the solenoid and target location were completed. Each of the collectors was biased to $70 \mathrm{~V}$ to collect the ion saturation signal. Each of the four cathodic arc plasma sources (CAPS) was driven with a 770 A discharge current. The final focus magnet was set to $8 \mathrm{~T}$, when it was operated.

When the FFS was not powered, the plasma signal was as high as $\geq 1 \times 10^{12} \mathrm{~cm}^{-3}$ between the FFS magnet and the target plane, and this density reached from $\leq 1 \times 10^{12} \mathrm{~cm}^{-3}$ to $\geq 1 \times 10^{11} \mathrm{~cm}^{-3}$ when it entered to the FFS. Figure 9(a) shows that all of the seven collectors, at the $\mathrm{x}$-axial coordinate, had a similar signal- except some variations in responses among probes that were due to the degree that the probe tips were not perfectly identical.

When the FFS was powered, the plasma forms a thin column of diameter $\sim 5 \mathrm{~mm}$ along the solenoid axis. The plasma signal was relatively low outside of this $\sim 5 \mathrm{~mm}$ diameter, as shown in Fig. $9(\mathrm{~b})$. It was inferred that particles entering the FFS with minimum angles passed through the axis to the downstream end of the FFS with their axial velocity $\left(\mathrm{v}_{0 \text { parallel }}=\mathrm{v}_{0} \cos \theta_{0}\right)$. Those particles with higher incident angles to the beam axis, $\mathrm{v}_{0 \text { transverse }}=\mathrm{v}_{0} \sin \theta_{0}$, reflected back from where the adjacent field lines were dense and developed a magnetic mirror, 
at the end of the FFS and off axis of the center line. Therefore, some of the reflected plasma increased the plasma density at the downstream end of the FFS as shown in the Fig. 9(b).

In order to convert these signals (plasma ion current) to plasma density, the calculated average velocities were considered for the two cases of the FFS (on or off), and for various axial locations. These calculated velocities were well matched with some measured velocities, measured using time-of-flight measurements. Due to the jitter in the CAPS pulse initiation we rather consider the calculated velocities to convert the plasma currents to densities. Figure 9(c) shows the plasma density at the center of the beam axis, measured using the center collector of the probe, when the FFS was on and off. A plasma density of $\geq 1 \times 10^{13} \mathrm{~cm}^{-3}$ was measured between the FFS magnet and the target plane, and this density approached $\geq 1 \times 10^{12} \mathrm{~cm}^{-3}$ at the entrance to the FFS. As mentioned earlier, this density was confined on the beam axis, within a diameter of $\approx 7 \mathrm{~mm}$, in the final focus solenoid when the solenoid was powered.

Figure 10 shows surface plots of the measured transverse and axial plasma distribution using the 37 collectors of the radial probe. The FFS was powered (8T), and the probe was driven from the upstream end of the FFS to the target plane. The maximum plasma electron density at the center of the FFS was $\geq 1 \times 10^{13} \mathrm{~cm}^{-3}$ for the measured plasma current of $53 \mathrm{~mA}$ for $\mathrm{Al}^{2+}$, using a velocity of $1.46 \times 10^{4} \mathrm{~m} / \mathrm{sec}$.

Figure 11 shows 3-D electrostatic PIC simulation, using the Warp code, of the four CAPS plasma density when the FFS is powered (8T). In this simulation $\mathrm{Al}^{+}$plasma, with velocity of 3 $\mathrm{cm} / \mu \mathrm{s}$, and temperature of $7 \mathrm{eV}$ was considered. Data was taken at $4.5 \mu \mathrm{s}$. This simulation shows qualitative agreement with the experiment. The density on axis shows similar double-humped profile, with a peak inside the solenoid. The density rapidly falls off when going off-axis inside the solenoid. Note that the peak density is only one tenths of experimentally determined value. This is likely due to underestimate of the injected plasma density coming from the four-CAPS.

Recently, a beam diameter of $2.5 \mathrm{~mm}$ (at FWHM) was measured when the system operated during a simultaneous radial and longitudinal compression experiment. Further measurements of plasma parameters, such as electron temperature or plasma pressure, and their effects on radial and longitudinal compression, will be addressed in future publications. 


\section{Conclusions}

We have successfully fabricated and operated four cathodic arc plasma sources, and the plasma was focused into a focusing solenoid with an $8 \mathrm{~T}$ field. A moveable plasma probe array with 37 collectors was developed to measure the radial and axial plasma distribution inside and outside of the solenoid. A plasma electron density of $\geq 1 \times 10^{13} \mathrm{~cm}^{-3}$ was achieved, and the plasma forms a thin column of diameter $\sim 5 \mathrm{~mm}$ along the solenoid axis when the FFS is powered.

\section{Acknowledgements:}

The authors are thankful to Professor Ron Davidson, Dr. Simon Yu, Dr. Alex Friedman, Dr. John Bernard, Dr. Enrique Henestroza, Dr. Igor Kaganovich, and Mr. Kevin Van Den Bogert for useful comment.

This work was supported by the U.S. Department of Energy under Contract No. DE-AC0205CH1 1231 with the Lawrence Berkeley National Laboratory, contract No. DE-AC52-07NA27344 with the Lawrence Livermore National Laboratory, and contract No. DE-AC02-76CH-O3073 with the Princeton Plasma Physics Laboratory. 


\section{References:}

[1] S. S. Yu et al., in Proc. of the 2003 Particle Accelerator Conf., edited by J.Chew, (IEEE, 2003), p.

98.

[2]B. G. Logan et al., Nucl. Fusion 45, 131 (2005).

[3] P. K. Roy et al., NIMA 544, 225 (2005).

[4] C. Thoma et al., Phys. Plasmas 12, 043102 (2005).

[5] R. C. Davidson et al., PRSTAB, 8, 064201 (2005).

[6] W. M. Sharp et al., NIMA 544, 398 (2005).

[7] W. M. Fawley et al., Phys. Plasmas 4, 880 (1997).

[8] D. R. Welch et al., NIMA 544, 236 (2005).

[9] C. Thoma et al., in Proc. of the PAC05, p.4006.

[10] P. K. Roy et al., PRL, 95, (2005), pp. 234801.

[11] A. B. Sefkow et al., NIMA 577, 289 (2007).

[12] P. A. Seidl et al., NIMA 577, 215 (2007).

[13] D. Welch et al., NIM A577, 231 (2007).

[14] J.E. Coleman et al., PRSTAB, 11, 050101 (2008).

[15] A. Anders et al., J. Appl. Phys. 91, 4824 (2002).

[16] A. Anders and R. A. MacGill, Surf. Coat. Technol. 133-134, 96 (2000).

[17] P.C. Efthimion et al., NIMA 577, 203 (2007)

[18] F. M. Bieniosek et al., in Proc. of the 2007 Particle Accelerator Conf. (IEEE catalog\# 07CH37866, USA, 2007), pp. 140-142.

[19] D. R. Welch et al., NIMA 464, 134 (2001). 
TABLE 1. Parameters of the final focus solenoid.

\begin{tabular}{|c|c|}
\hline Parameter & $\overline{\text { Value }}$ \\
\hline Maximum Solenoid field: & $8 \mathrm{~T}$ \\
\hline Effective field length: & $10.15 \mathrm{~cm}$ \\
\hline Physical length of the solenoid & $9 \mathrm{~cm}$ \\
\hline $\begin{array}{l}\text { Winding pack ID and OD } \\
\mathrm{cm}\end{array}$ & $5.1 \mathrm{~cm}$ and 6.7 \\
\hline $\begin{array}{l}\text { Wire specification: } 24 \text { stra } \\
\text { heavy build insulation } \mathrm{MW} 80 \\
\text { to } 1 \mathrm{~cm} \times 0.16 \mathrm{~cm} \text {, wrapped in } \mathrm{N}\end{array}$ & $\begin{array}{l}\text { nd gauge } 20 \text { AWG, } \\
155 \text { Deg., profiled } \\
\text { omex paper }\end{array}$ \\
\hline Number of turns: & 32 (8 turns/layer) \\
\hline Coil resistance: & $7.75 \times 10^{-3} \Omega$ \\
\hline Coil Inductance: & $2.80 \times 10^{-5} \mathrm{H}$. \\
\hline Epoxy specification: & CTD-101K \\
\hline Stored energy: & $4.5 \mathrm{~kJ}$ \\
\hline Max. voltage across magnet: & $2.9 \mathrm{kV}$ \\
\hline Max. current: & $21.7 \mathrm{kA}$ \\
\hline Pulse length : & $837 \mu \mathrm{s}$ \\
\hline
\end{tabular}




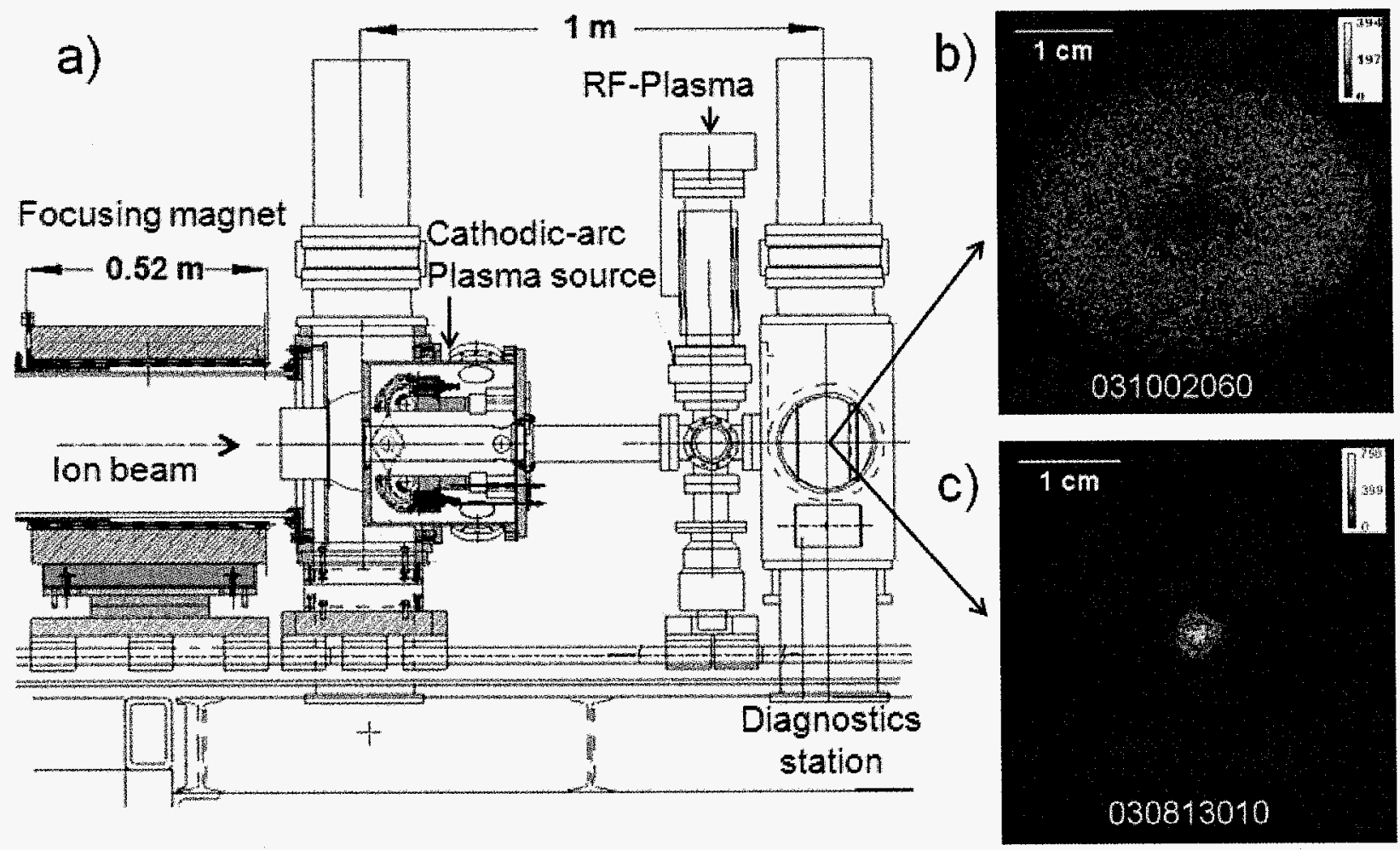

Fig.1. (a) Schematic of the beam neutralization section with two filtered cathodic arc plasma sources (FCAPS); (b) beam image at the focal plane without plasma neutralization; and (c) with plasma neutralization. 


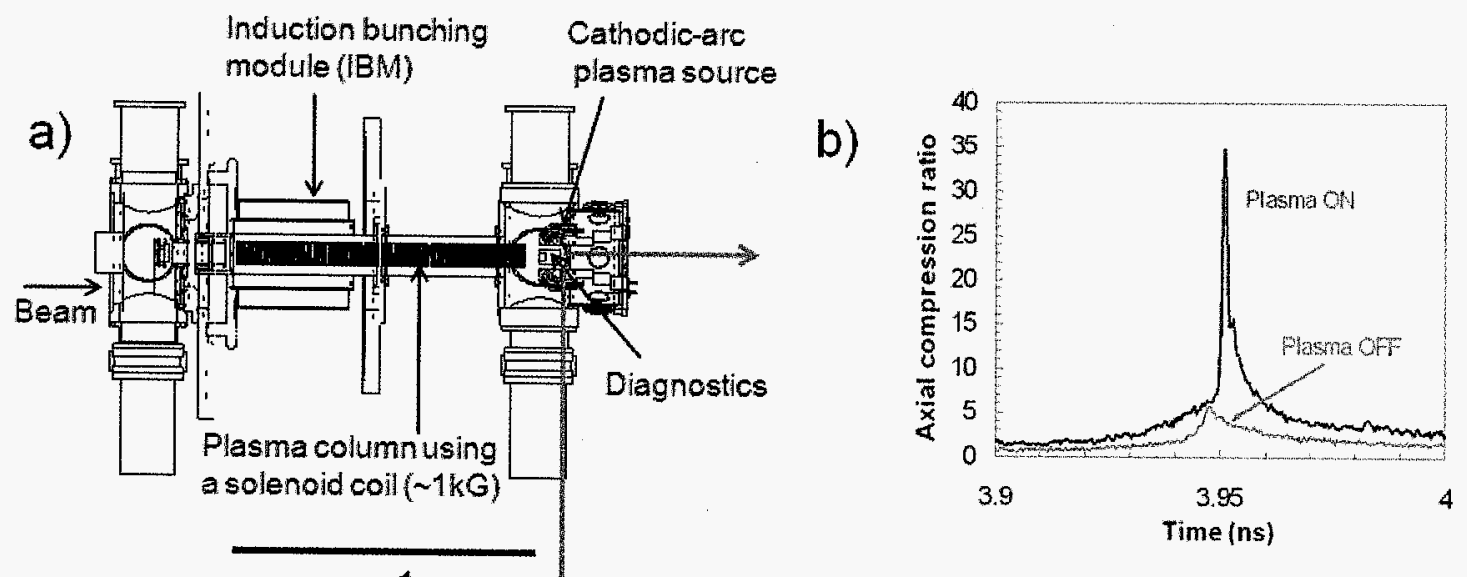

c)

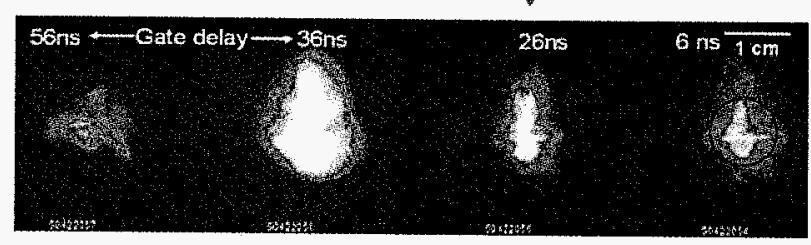

d)

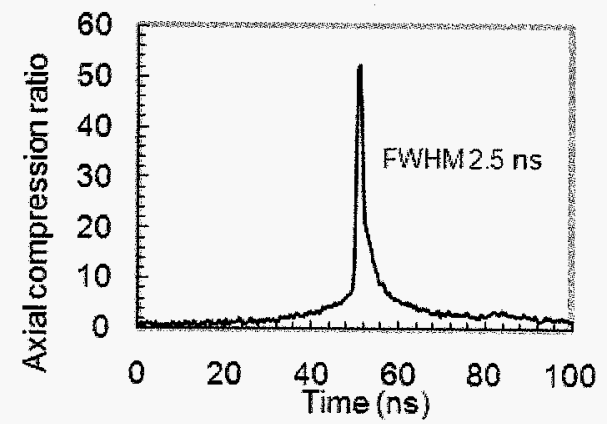

Fig.2. Initial NDCX-I experiment: (a) schematic of the plasma column. Plasma drifted from the downstream end to the IBM using a 80-cm-long solenoid coil; (b) an example of axial compression with and without background plasma; (c) beam profile ( $>6 \mathrm{~mm}$ FWHM) at the point of maximum axial compression; and (d) compression ratio obtained from the phototube diagnostic. 

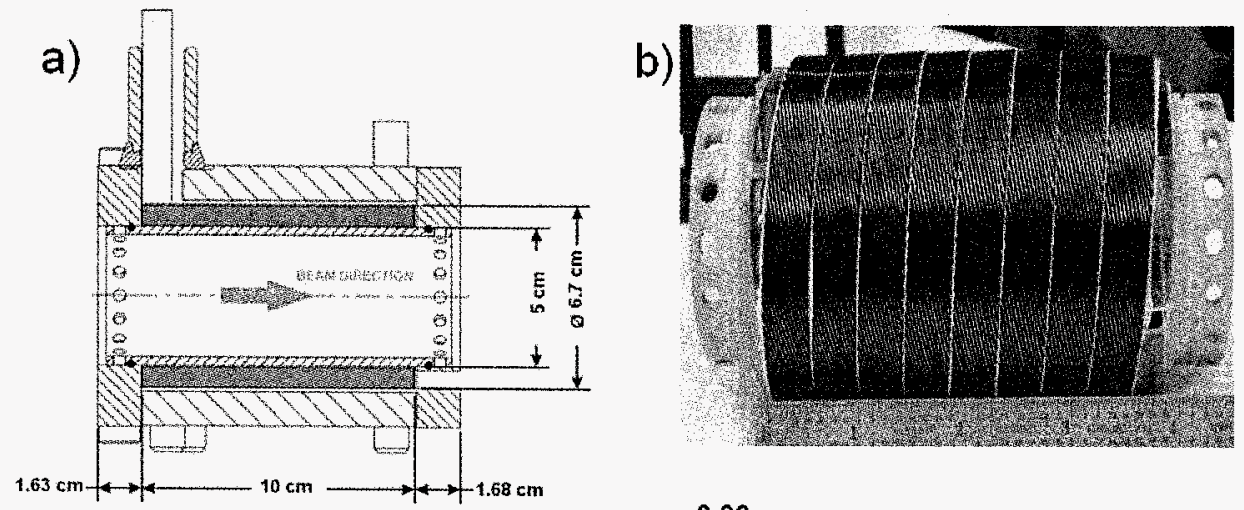

c)
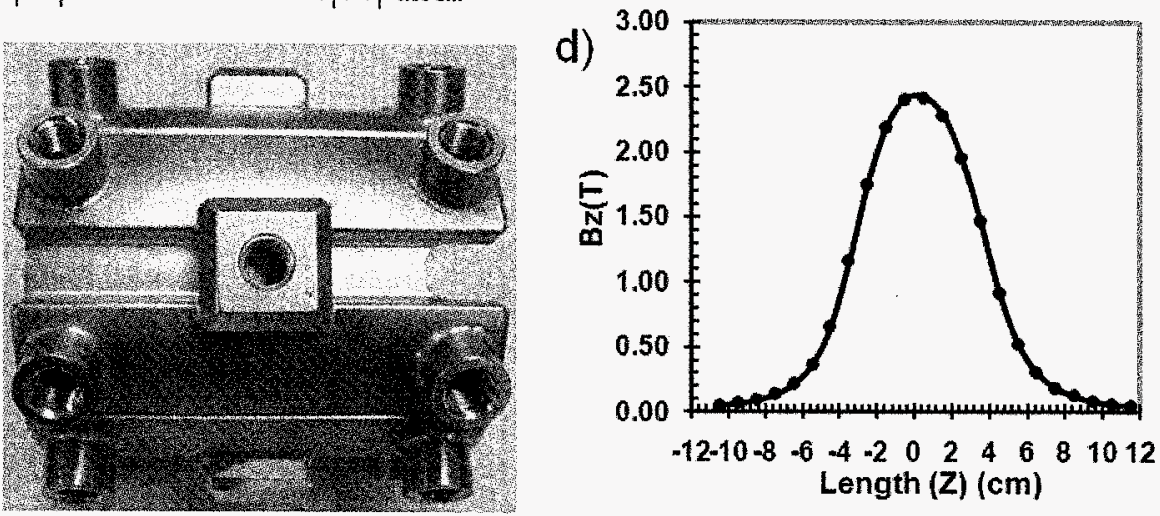

Fig. 3. (a) Schematic final focus solenoid; (b) winding coil; (c) coil cooling jack; and (d) an example of the characteristic magnetic field profile. 


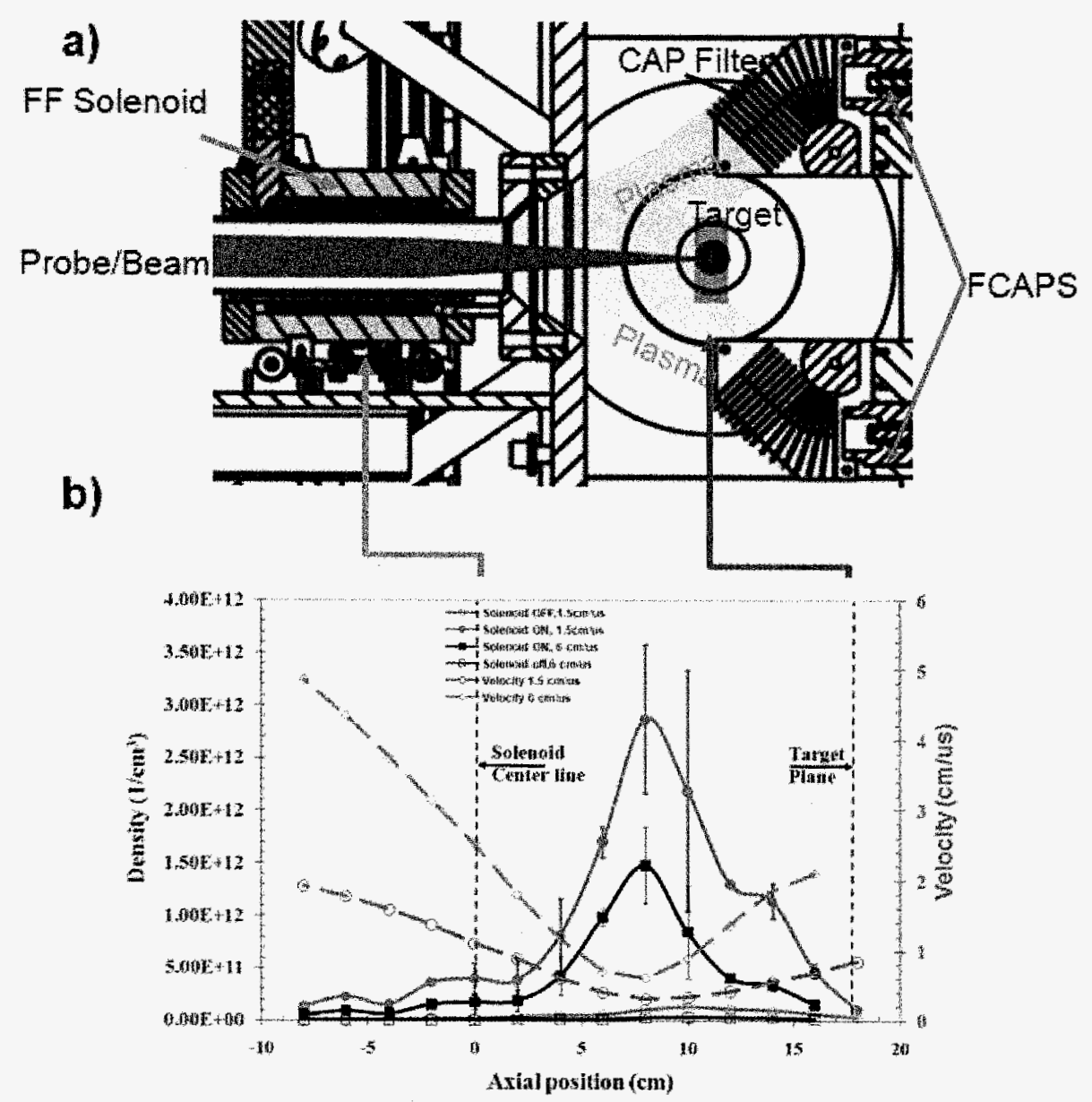

Fig. 4. (a) Schematic of the FCAPS and the FFS in the target chamber; and (b) inferred experimental axial plasma density distributions with and without a magnetic field, for two initial velocities in the simulations ( $1 \mathrm{kV}$ discharge). The dotted lines (blue in color) represent calculated velocities, initially at $1.5 \mathrm{~cm} / \mu \mathrm{s}$ and $6 \mathrm{~cm} / \mu \mathrm{s}$. 



Figure 5: (a) Schematic of the integration of the final focus solenoid (FFS), two-FCAPS and FEPS assembly with a $\mathrm{K}^{+}$NDCX beam line (IBM: $25 \mathrm{kV}$, FCAPS $\sim 470$ A, FEP: $9 \mathrm{kV}$ ); (b) compressed beam image with the final focus solenoid powered (5T); (c) the FFS without power; and (d) profiles of the two images. 
a)

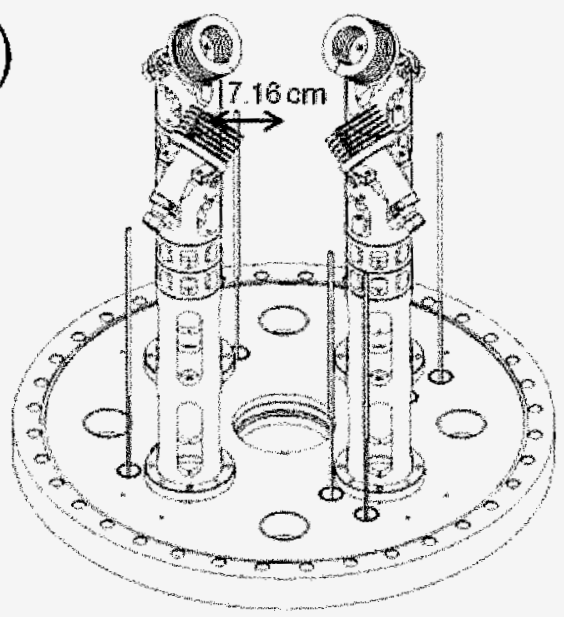

c)

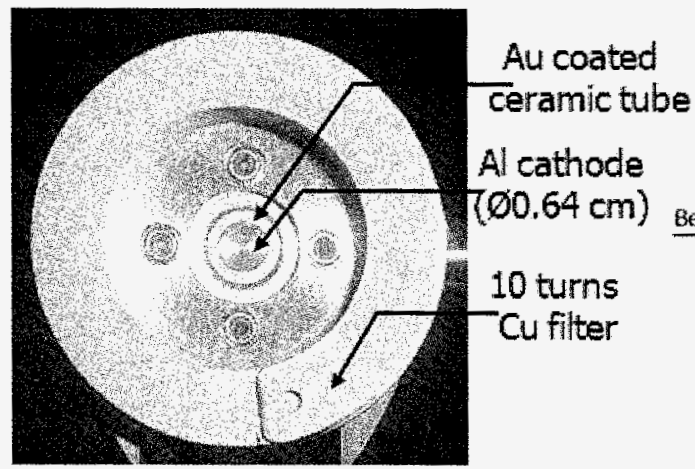

b)
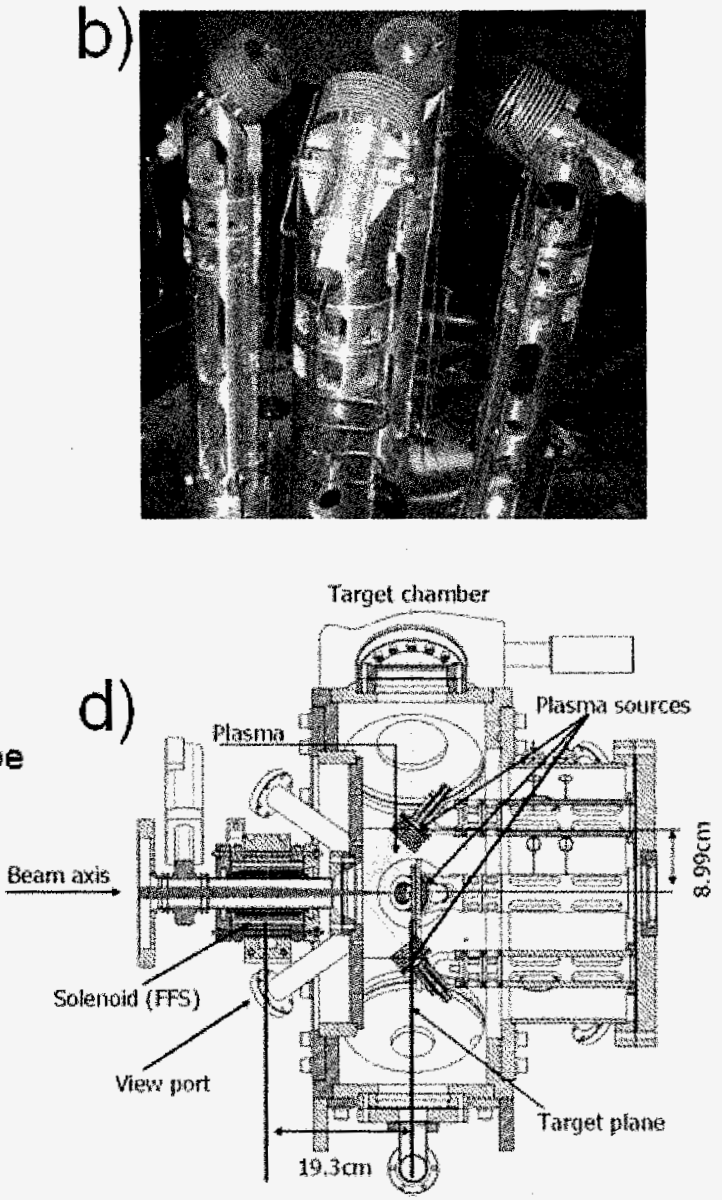

Fig. 6. (a) Schematic of the four filtered cathodic arc plasma sources; (b) photograph of the CAPS; (c) photograph of the cathode and filter; and (d) a sketch of the target chamber with four cathodic are plasma sources and the final focus solenoid (FFS). 
a)
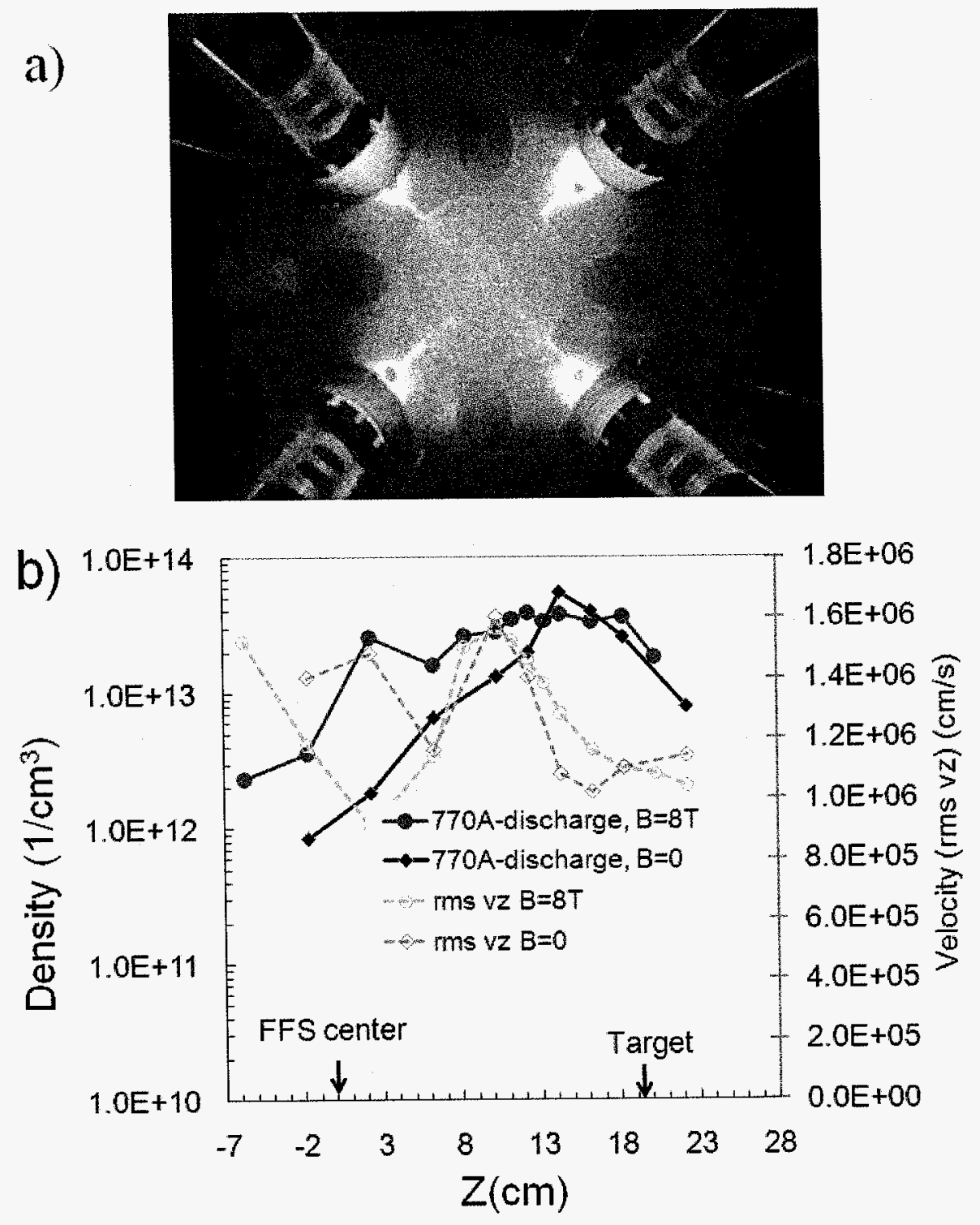

Fig.7. (a) Flash of the four-CAPS; and (b) axial plasma density and rms velocity (770A discharge with each of the four CAPS and $8 \mathrm{~T}$ field with the FFS when operated, area of the probe tip was $1.46 \mathrm{~mm}^{2}$ ). 

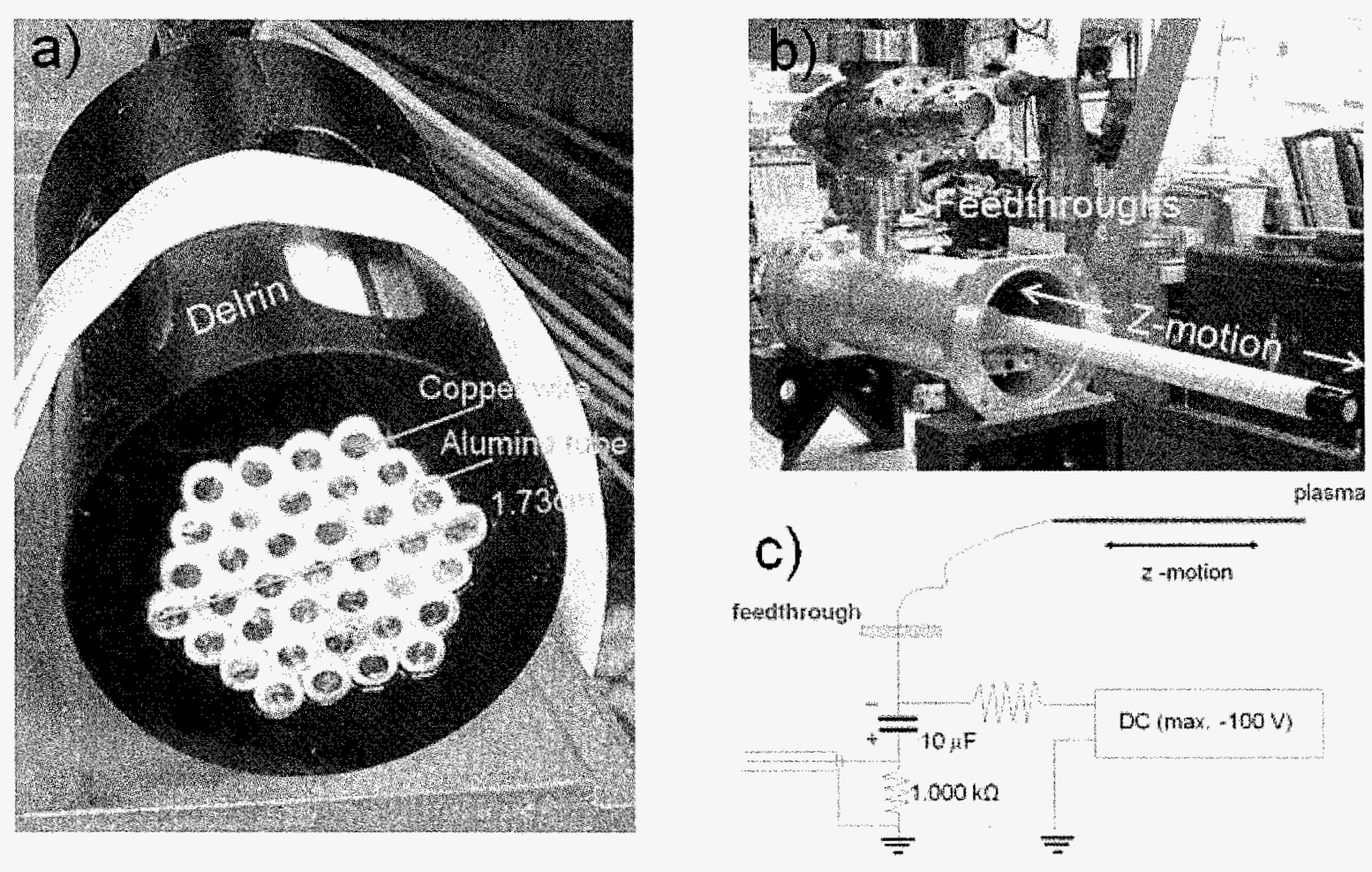

Fig. 8. (a) 37-collectors probe array, each probe is $1.6 \mathrm{~mm}$ in diameter, with a collective diameter of 1.73 $\mathrm{cm}$; (b) axial drive unit of the probe and its feedthroughs; and (c) circuit diagram of the collector bias, each collector was biased to $70 \mathrm{~V}$ for operation in the ion saturation mode. 

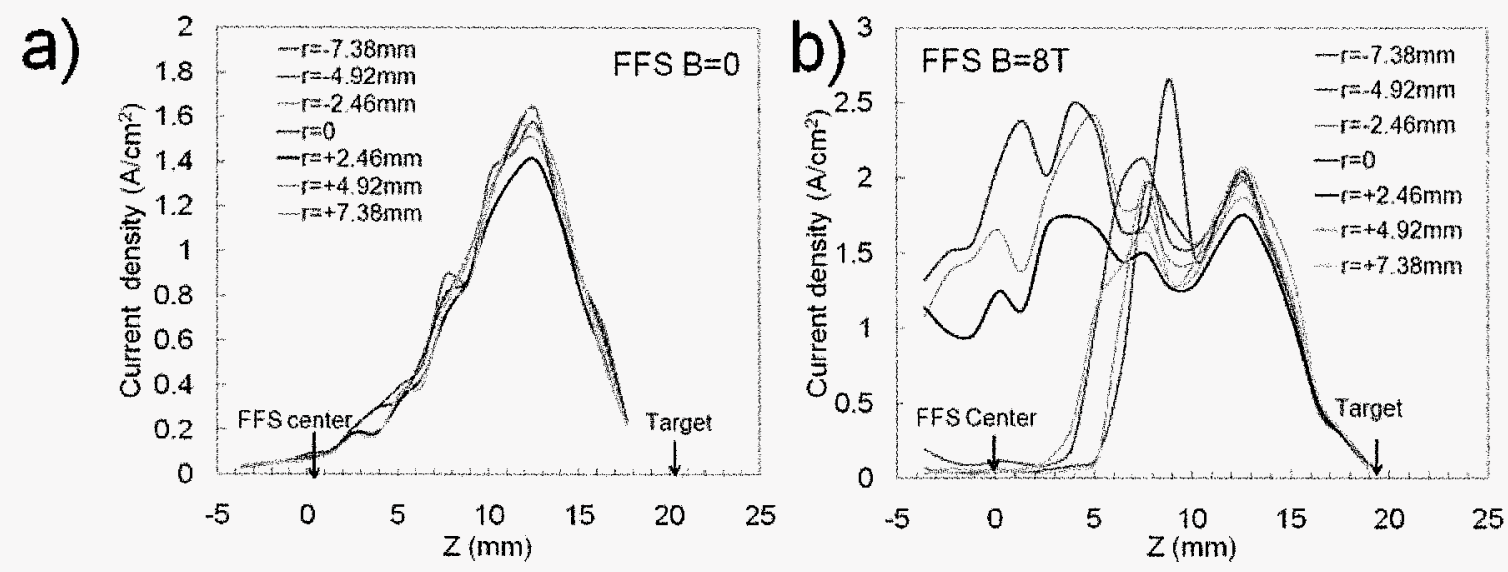

C)

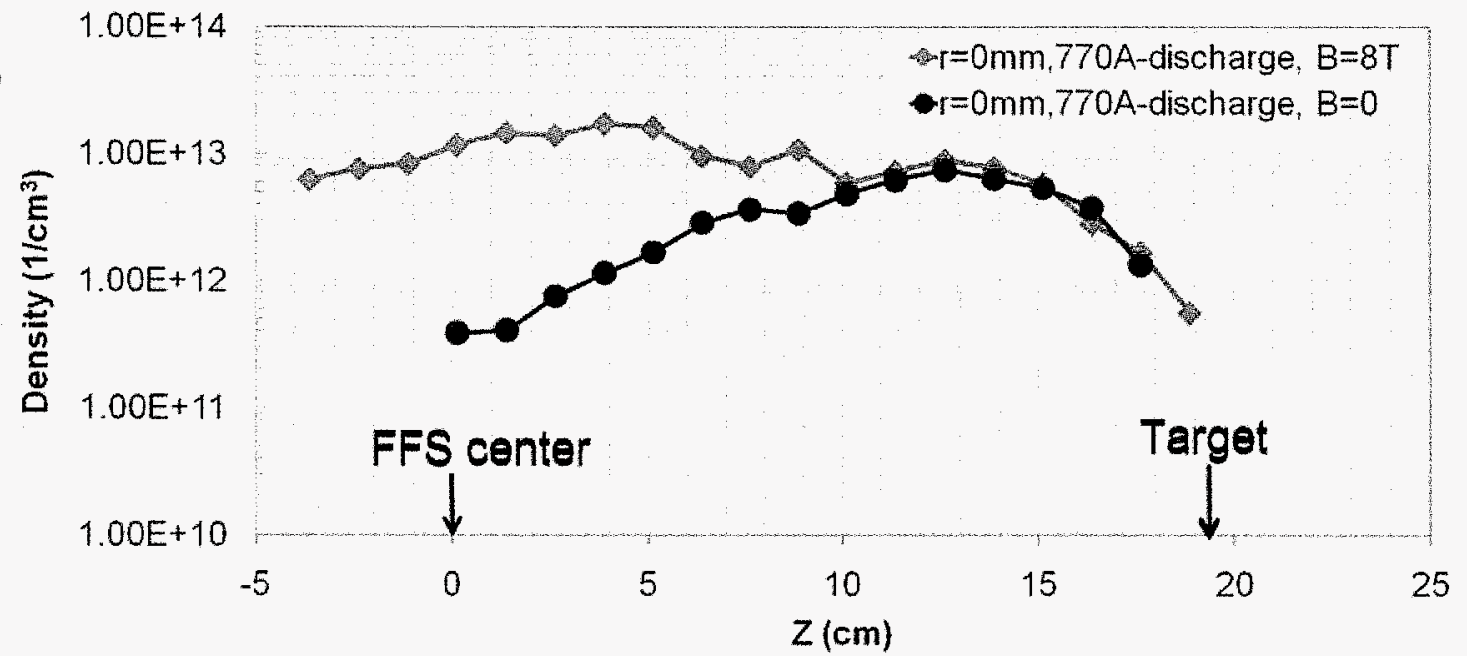

Fig.9. (a) Radial plasma ion current signals with respect to the z- axis for the center seven collectors of the probe array when the FFS was not powered, (b) the collectors signals when the FFS was powered (8T); and (c) axial plasma density measured using only the center collector, when the FFS was powered (line with solid diamonds) and not powered (line with solid circles). 
Up stream $\leftarrow$ FFS $\rightarrow$ Down stream

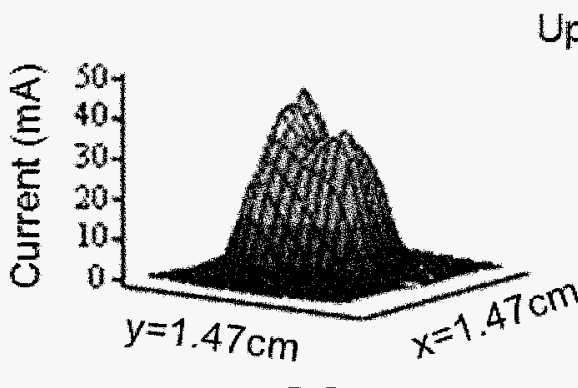

$-2.3 \mathrm{~cm}$

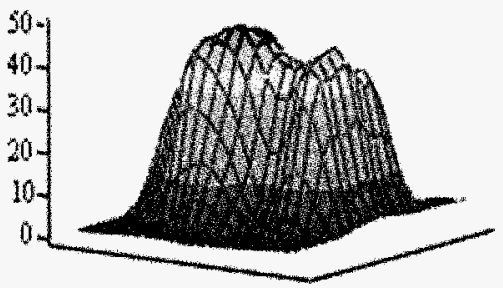

$5.1 \mathrm{~cm}$

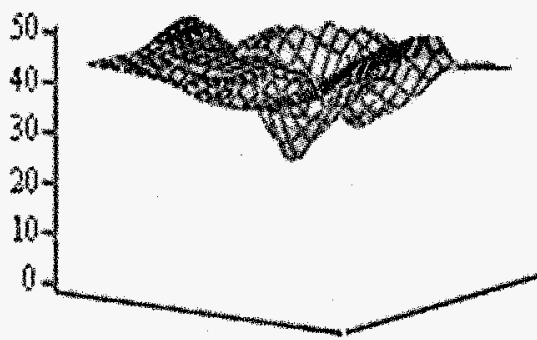

$12.6 \mathrm{~cm}$

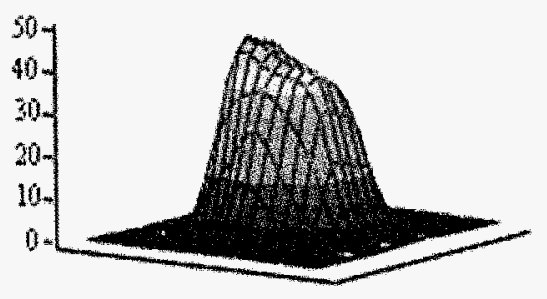

$0.14 \mathrm{~cm}$
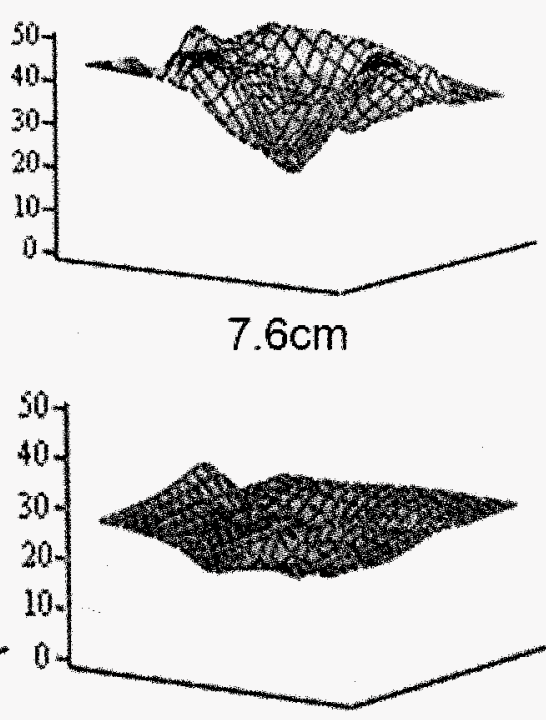

$15.1 \mathrm{~cm}$

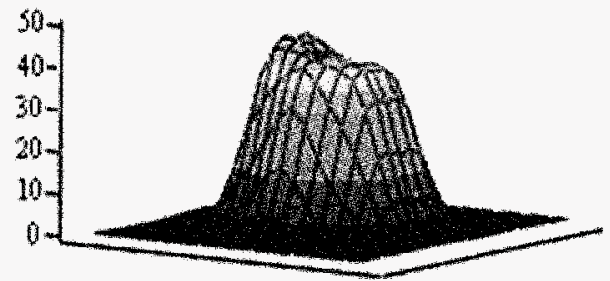

$2.6 \mathrm{~cm}$
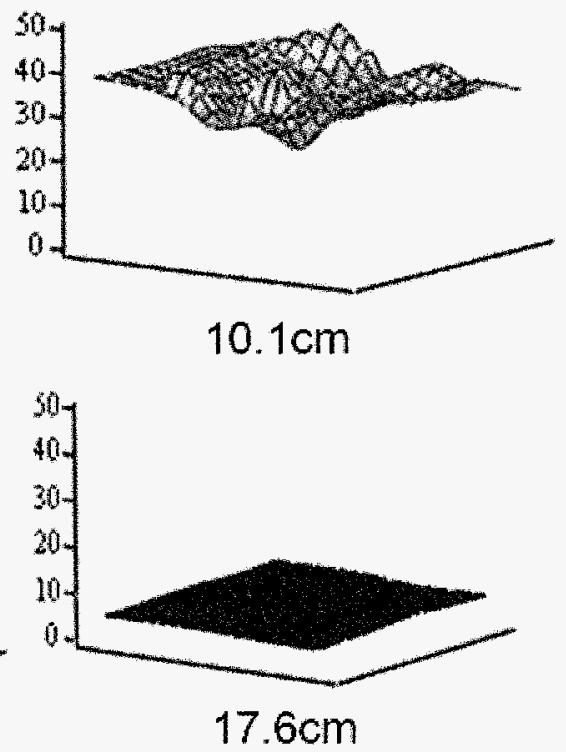

Fig.10. Surface plots of the measured (using the 37 collectors) transverse and axial plasma distribution when the FFS and the four-CAPS were operated with $8 \mathrm{~T}$ and $770 \mathrm{~A}$, respectively. The probe was driven from the upstream end of the FFS to the target plane. 


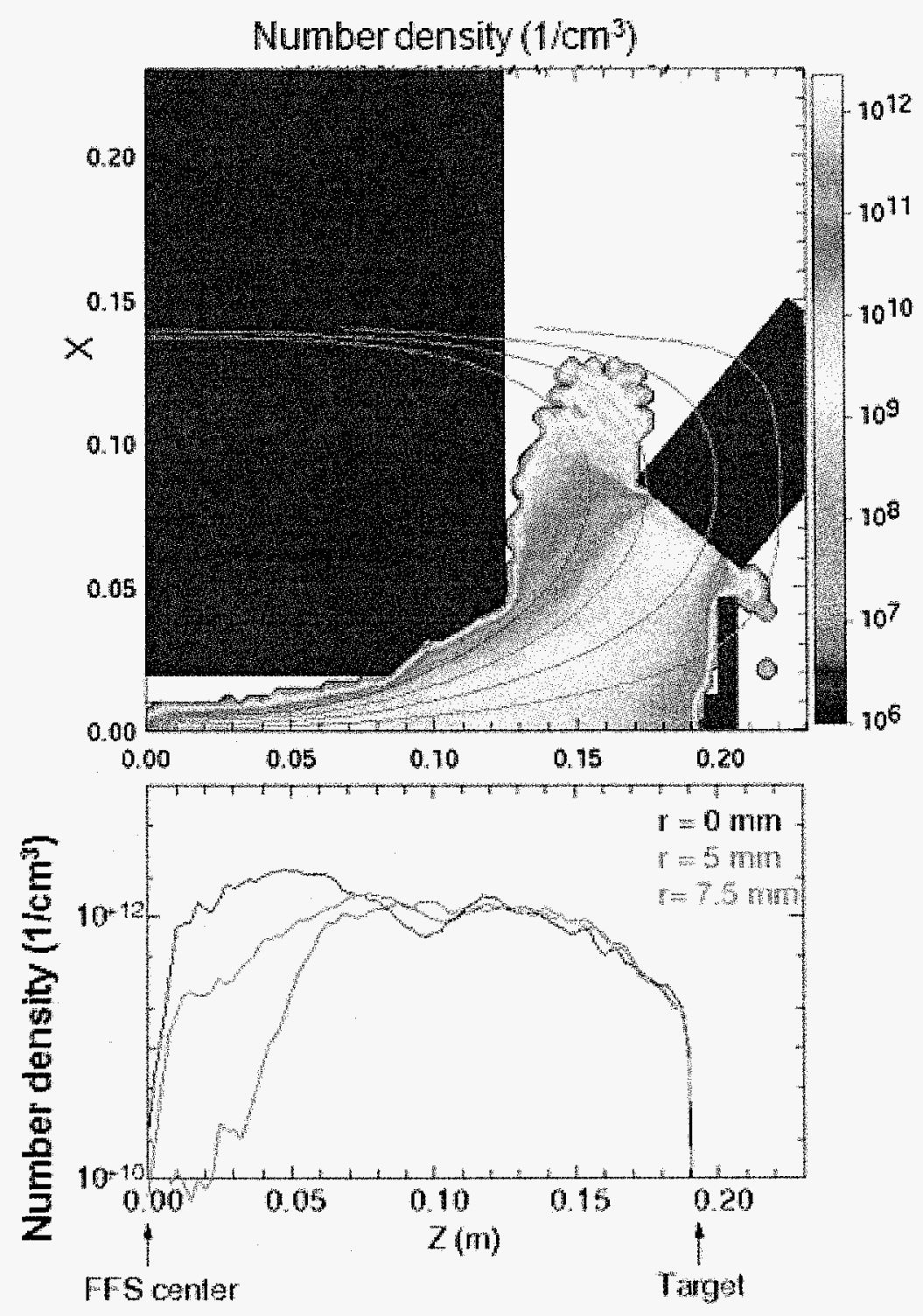

Fig. 11. 3-D electrostatic PIC simulation of the four-CAP plasma density when the FFS is powered (8T). 